\title{
Overtraining and extradimensional shift learning by cats
}

\author{
J. M. WARREN \\ Pennsylvania State University, UniversityPark, Pennsylvania 16802
}

\begin{abstract}
Twenty-four cats were trained with stimuli that presented uncorrelated differences in size and brightness, on discrimination and extradimensional shift (EDS) problems in a Grice box. The cue relevant in EDS training was present and irrelevant in initial discrimination training, and the cue relevant in discrimination training was present and irrelevant in EDS training. Cats overtrained for 144 trials in discrimination training learned the EDS as quickly as animals switched to EDS without overtraining. This result is hard to reconcile with Sutherland and Mackintosh's attention theory of discrimination learning by animals.
\end{abstract}

If learning to attend to the relevant stimulus dimension is critical in discrimination learning by animals, and if overtraining (OT) selectively strengthens attention to the momentarily relevant dimension, OT should retard the solution of an extradimensional shift (EDS) problem. A previously irrelevant cue should become relevant and a previously relevant cue should be irrelevant (Mackintosh, 1974; Sutherland \& Mackintosh, 1971).

Experiments with rats have yielded somewhat inconsistent support for this prediction, but suggest that three factors are likely to produce slower EDS learning by overtrained animals. OT is likely to retard EDS learning if (1) the formerly relevant cue is present and irrelevant in the EDS problem, (2) the apparatus is a jumping stand or Grice box, (3) the stimulus cues used in EDS training are not readily discriminated (Sutherland and Mackintosh, 1971).

The purpose of this experiment was to determine if overtrained cats are retarded in EDS learning when tested under the circumstances that Sutherland and Mackintosh (1971) specified are most likely to produce the effect.

\section{METHOD}

\section{Subjects}

The subjects were 24 adult mongrel cats that had learned a single shape discrimination with no irrelevant visual cues in the Grice box (Warren, 1976). They were tested under 16 to $23 \mathrm{~h}$ of food deprivation and were reinforced for correct choices with a 6-mm cube of pork kidney or an equal volume of tuna fish.

\section{Apparatus}

The Grice box was $37 \mathrm{~cm}$ high and painted gray throughout. A $28 \times 56 \mathrm{~cm}$ startbox, with opaque and transparent doors, opened into a choice area, $41 \mathrm{~cm}$ deep, beyond which were two $31 \times 44 \mathrm{~cm}$ response compartments.

The stimuli were vertically oriented wooden objects, black and white squares with areas of 49 and $81 \mathrm{~cm}^{2}$. The black (B) and white (W), large (L) and small (S) stimuli were always combined in pairs that presented differences in both brightness and size (LW vs. SB and SW vs. LB). Each of these pairs was presented on half the trials in a test session in a balanced irregu- lar sequence; the position of the rewarded stimulus value varied over trials according to an independent irregular sequence.

\section{Procedure}

Adaptation and preference tests. The cats were initially shaped with visible kidney or tuna on both sides of the apparatus until they responded quickly enough to complete 24 trials in $10 \mathrm{~min}$. They were then observed on 24 trials with the stimulus pairs and the position of the members of each pair varied as in discrimination training but with nondifferential reinforcement. Preferences manifested on this test were used to assign cats to groups, equated for initial preference, for training on the size and brightness discrimination tasks.

Discrimination and transfer training. Twelve cats were first trained to discriminate brightness and 12 to discriminate size, with 24 trials a day to a criterion of 44 correct responses in 48 noncorrection trials $(92 \%)$ over 2 consecutive days. Equated groups were then given 144 or no overtraining trials before training on the EDS problem. The cats initially trained on brightness with size irrelevant then had to learn to discriminate size with brightness cues present and irrelevant; the subjects originally trained on size with brightness irrelevant were obliged to discriminate brightness with size cues present and irrelevant in the EDS. The animals continued to receive 24 trials a day and were trained to the criterion of 44 correct in 48 trials on the EDS task.

\section{RESULTS}

Mean errors to criterion on the size and brightness discriminations are presented in Table 1 for three groups of cats, those that learned each task without prior training (the naive groups) and those that learned each discrimination as an EDS after overtraining or training only to criterion on the other dimension. Inspection of

Table 1

Errors to Criterion in Discrimination Learning

\begin{tabular}{lcrlcc} 
& \multicolumn{2}{c}{ Size } & & \multicolumn{2}{c}{ Brightness } \\
\cline { 2 - 3 } \cline { 6 - 6 } Pretraining & Mean & SD & & Mean & SD \\
\hline None & 191 & 112 & & 57 & 23 \\
Criterion & 179 & 51 & & 49 & 27 \\
Overtrained & 155 & 76 & & 43 & 19 \\
\hline
\end{tabular}


Table 1 reveals that the overtrained cats made somewhat fewer errors than the naive animals (19\% on size, $25 \%$ on brightness), and the scores for the cats trained only to criterion in original learning were intermediate between those of the overtrained and naive subjects. It is also apparent that there is considerable variation in error scores within groups.

Analyses of variance on the error scores for size and for brightness discrimination yielded nonsignificant values of $F$, both less than 1 . Since the within-groups variance was large, all comparisons between pairs of groups were evaluated by Mann-Whitney U-tests; the lowest $p$ value yielded by the U-tests was .35 . Thus, the data of this experiment indicate that overtraining neither significantly facilitates nor significantly retards subsequent learning of an EDS problem by cats.

\section{DISCUSSION}

Retardation, facilitation, and no effect have been observed in EDS learning by rats overtrained on their initial discrimination task. Sutherland and Mackintosh (1971) attributed the theoretically troublesome cases of facilitation or no effect on EDS after OT to three experimental conditions. OT is not expected to depress EDS performance if: (1) the EDS task is too easy (e.g., Sutherland \& Andelman, 1969); (2) the cue that is present in the first stage of training is not present and irrelevant in the EDS problem (e.g., Waller, 1970); (3) the apparatus does not optimize orientation to relevant cues (e.g., Siegel, 1967).

This experiment with cats was designed to incorporate the features most favorable, in theory, for OT to depress EDS learning in rats and presumably other mammals. (1) Salient (brightness) and nonsalient (size) cues were used in EDS training, to see if the effects of OT on EDS vary with stimulus conspicuity. (2) The cues that were relevant in initial discrimination training were retained and irrelevant in the EDS. (3) The
Grice box is an apparatus that Sutherland and Mackintosh judged as favorable to retardation of EDS learning by overtrained rats. Yet no evidence that OT slows the rate of EDS learning by cats was obtained.

Hall (1974) found that rats overtrained on a discrimination between oblique lines learned a black-white discrimination as quickly as did control subjects but were superior to controls on the reversal of the brightness discrimination. Since OT is supposed to strengthen attention to relevant stimulus dimensions, OT should retard EDS and facilitate reversal learning. Hall's dissociation of the two predicted effects in the same animals, tested in the same apparatus, implies that OT influences EDS and reversal learning through different mechanisms.

It seems clear that the crucial conditions for retardation of EDS learning by OT have not yet been completely specified; the effect of OT on EDS learning is too fragile and capricious to provide strong support for an attention theory of discrimination learning.

\section{REFERENCES}

HALL, G. Transfer effects produced by overtraining in the rat. Journal of Comparative and Physiological Psychology, 1974, 87, 938-944.

MACKInTosh, N. J. The psychology of animal learning. London: Academic Press, 1974.

SIEGEL, S. Overtraining and transfer processes. Journal of Comparative and Physiological Psychology, 1967, 64, 471-477.

Sutherland, N. S., \& ANDElman, L. Effects of overtraining on intra- and extra-dimensional shifts. Psychonomic Science, 1969, 15, 253-254.

Sutherland, N. S., \& Mackintosh, J. J. Mechanisms of animal discrimination learning. New York: Academic Press, 1971.

WALlER, T. G. Facilitation of an extradimensional shift with overtraining in rats. Psychonomic Science, 1970, 20, 172-174.

WARREN, J. M. Irrelevant cues and shape discrimination learning by cats. Animal Learning \& Behavior, 1976, 4, 22-24.

(Received for publication May 30, 1978.) 